\title{
Minimizing bone gaps when using custom pediatric cranial implants is associated with implant success
}

\author{
${ }^{*}$ Christian A. Bowers, MD, Jaron H. McMullin, MD, ${ }^{2}$ Cameron Brimley, MS, ${ }^{3}$ \\ Linsey Etherington, MD, ${ }^{2}$ Faizi A. Siddiqi, MD, ${ }^{2}$ and Jay Riva-Cambrin, MD, MSc ${ }^{1}$ \\ Departments of ${ }^{1}$ Neurosurgery and ${ }^{2}$ Plastic and Reconstructive Surgery, Primary Children's Medical Center, University of Utah; \\ and ${ }^{3}$ University of Utah School of Medicine, Salt Lake City, Utah
}

\begin{abstract}
OBJECT Occasionally after a craniotomy, the bone flap is discarded (as in the case of osteomyelitis) or is resorbed (especially after trauma), and an artificial implant must be inserted in a delayed fashion. Polyetheretherketone (PEEK) implants and hard-tissue replacement patient-matched implants (HTR-PMI) are both commonly used in such cases. This study sought to compare the failure rate of these 2 implants and identify risk factors of artificial implant failure in pediatric patients.

METHODS This was a retrospective cohort study examining all pediatric patients who received PEEK or HTR-PMI cranioplasty implants from 2000 to 2013 at a single institution. The authors examined the following variables: age, sex, race, mechanism, surgeon, posttraumatic hydrocephalus, time to cranioplasty, bone gap width, and implant type. The primary outcome of interest was implant failure, defined as subsequent removal and replacement of the implant. These variables were analyzed in a bivariate statistical fashion and in a multivariate logistic regression model for the significant variables.

RESULTS The authors found that $78.3 \%$ (54/69) of implants were successful. The mean patient age was 8.2 years, and a majority of patients were male $(73 \%, 50 / 69)$; the mean follow-up for the cohort was 33.3 months. The success rate of the 41 HTR-PMI implants was $78.1 \%$, and the success rate of the 28 PEEK implants was $78.6 \%$ ( $p=0.96)$. Implants with a bone gap of $>6 \mathrm{~mm}$ were successful in $33.3 \%$ of cases, whereas implants with a gap of $<6 \mathrm{~mm}$ had a success rate of $82.5 \%(p=0.02)$. In a multivariate model with custom-type implants, previous failed custom cranial implants, time elapsed from previous cranioplasty attempt, and bone gap size, the only independent risk factor for implant failure was a bone gap > $6 \mathrm{~mm}$ (odds ratio 8.3, 95\% confidence interval 1.2-55.9).
\end{abstract}

CONCLUSIONS PEEK and HTR-PMI implants appear to be equally successful when custom implantation is required. A bone gap of $>6 \mathrm{~mm}$ with a custom implant in children results in significantly higher artificial implant failure.

http://thejns.org/doi/abs/10.3171/2015.2.PEDS14536

KEY WORDS cranioplasty; custom cranial implants; failure rates

$\mathrm{S}$ kULL trephinations and subsequent cranioplasties have been performed throughout human history, with the first evidence of cranioplasty dating as far back as $2000 \mathrm{BC}$ and the first modern report of successful cranioplasty dating to $1668{ }^{2,11}$ In the past 400 years, the substances used in cranioplasties have included cork, rubber, metals, xenografts, autologous bone, silicone, and coconut shell., ${ }^{2,11}$ The search for the ideal cranioplasty material continues to this day.
Currently, 2 types of custom cranioplasty implants are frequently used for pediatric cranioplasties: polyetheretherketone (PEEK) and hard-tissue replacement patientmatched implants (HTR-PMIs). The cranioplasty results with these materials have not been extensively studied. In the last 15 years, 3 series have described the results of using PEEK implants or HTR-PMIs. In the literature describing the use of these implants, the case reports or series have been small ( $<40$ total patients), retrospective, composed

ABBREVIATIONS $\mathrm{Cl}$ = confidence interval; HTR-PMI = hard-tissue replacement patient-matched implant; PEEK = polyetheretherketone.

SUBMITTED October 3, 2014. ACCEPTED February 20, 2015.

INCLUDE WHEN CITING Published online July 10, 2015; DOI: 10.3171/2015.2.PEDS14536.

DISCLOSURE The authors report no conflict of interest concerning the materials or methods used in this study or the findings specified in this paper.

* Drs. Bowers and McMullin contributed equally to this work. 
of adult patients, and limited in follow-up., ${ }^{5,14}$ Although the first few studies reported complete success with no complications, the largest and most recent series created doubt regarding the true success rate of these implants, because a $23.8 \%$ complication rate was documented. ${ }^{10}$

In general, we have considered these custom cranial implants to have high success rates as demonstrated in our recent publication examining risk factors of bone flap resorption following hemicraniectomy for pediatric traumatic brain injury. We found that age $<2.5$ years old, hydrocephalus, an underlying cerebral contusion, and a comminuted skull fracture were all independent risk factors for bone flap resorption and that custom cranioplasties were successful in revision cranioplasty following bone flap resorption in $91.7 \%$ (11/12) of the cases. ${ }^{1}$ In this current study, our goal was to identify whether either cranioplasty implant type, PEEK or HTR-PMI, was more successful than the other and to identify risk factors of custom cranial implant failure. ${ }^{1}$

\section{Methods \\ Patient Sample}

We performed a retrospective cohort chart review to identify patients $<18$ years old with cranial defects necessitating cranioplasty with an HTR-PMI or PEEK implant from January 1, 2000, through December 31, 2012, at Primary Children's Hospital, University of Utah. Standard cranial titanium plating systems were used for flap attachment in all patients. Drains were used sparingly, because of increased risk of infection and because one surgeon used them more often. This surgeon's preference was taken into account when we analyzed the different attending surgeons. Patients were excluded if craniosynostosis was the cause of their cranial defect, because these small cranial defects are distinct from the large frontotemporoparietal cranial defects typically seen after decompressive craniectomy, infected bone flaps, or cranial defects created from tumor resection involving the skull. Patients were also excluded if any other cranioplasty material was used besides PEEK or HTR-PMI or if the cranial defect was in the posterior fossa or an occipital location, because PEEK implants and HTR-PMIs are rarely indicated for these types of defects.

\section{Clinical Variables}

Each variable was clearly defined a priori before chart abstraction to minimize measurement bias. Clinical variables examined included sex, race, age at implantation, and the cause of the cranial defect (including trauma, infected craniotomy flap, tumor-involved bone, and "other etiologies"). Other etiologies included defects secondary to craniosynostosis defect repair, decompressive hemicraniectomy for stroke, or craniotomies for vascular lesions or tumors that had subsequent bone flap resorption or were discarded. Other clinical variables studied included attending surgeon, involvement of the plastic surgery team, previous autologous cranioplasty failure, previous infection with the length of the quiescent period free of infection, previous PEEK or HTR-PMI cranioplasty failures, and length of follow-up.

\section{Surgical Variables}

The implant type used, PEEK or HTR-PMI, was the key variable to address the question of superiority between the 2 custom implant types. The extent of the gap between the implant and the native frontal bone was also measured on the postoperative CT head and subsequent CT scans. Any defect left along the floor of the temporal bone was not included in the measurement. At our hospital for most of the years of the study, CT scans were only acquired in the axial plane. The frontal area is easily visualized and measurable on an axial CT scan, and this allowed for a consistent measurement of the medial-lateral bone gap on serial imaging; however, the flap site of attachment for the temporal bone is vertical and therefore is less reliably measured while frequent large gaps are left in this noncosmetic region. Because of the observed trend in the association between increased bone gap width and implant failure, we examined it more closely. The data appeared to self-dichotomize with a cutoff of $6 \mathrm{~mm}$, leading us to choose this cutoff point. We used an iterative process, with gap cutoffs close to $6 \mathrm{~mm}$ (i.e., $4 \mathrm{~mm}, 5 \mathrm{~mm}$, $6 \mathrm{~mm}, 7 \mathrm{~mm}$, and $8 \mathrm{~mm}$ ), and $6 \mathrm{~mm}$ still appeared to be representative of the data.

\section{Outcome}

The primary outcome was implant failure, defined as any subsequent surgery performed to remove the cranioplasty implant, replacing it with a new implant because of infection, dislodgement, or unacceptable cosmesis. All chart reviews and data acquisition were in compliance with the regulations determined by the University of Utah Institutional Review Board and with the Health Insurance Portability and Accountability Act (HIPAA) of 1996.

\section{Statistical Analysis}

All statistical analyses were conducted using SAS version 9.3 (SAS Institute). Each variable was compared with the outcome, using the chi-square test for categorical variables and the Student $t$-test for continuous variables. All variables were tested and found to be parametric. Any variables found to be significant $(p<0.05)$ or nearly significant $(\mathrm{p}<0.10)$ were included in the multivariate analysis. Stepwise logistic regression was used to determine which of those variables were independently associated ( $p$ $<0.05$ ) with implant failure.

\section{Results \\ Patient Sample}

A total of 54 children were identified as having undergone 69 custom cranioplasties (50 in boys, 19 in girls; mean age 8.2 years). Each procedure was treated as an independent event for analysis of cranial defects with PEEK implants or HTR-PMIs from 2000 through 2012 at our institution. There were 15 implant failures for a failure rate of $21.7 \%$.

In the vast majority of cranioplasty procedures, the patient had cranial defects from prior trauma (41 cases, 59.4\%; Table 1). Previous tumor resections involving the bone and resulting in cranial defects only accounted for 6 implants (11.1\%), while a previous infected flap accounted 
TABLE 1. Demographic, clinical, and surgical characteristics of the overall cohort*

\begin{tabular}{|c|c|}
\hline Variable & Value \\
\hline Procedural events in 54 patients & 69 \\
\hline Mean age at presentation in yrs \pm SD & $8.2 \pm 5.5$ \\
\hline Males & $50(72.5)$ \\
\hline Caucasian race & $61(88.4)$ \\
\hline \multicolumn{2}{|l|}{ Etiology } \\
\hline Trauma & $41(59.4)$ \\
\hline Infected craniotomy flap & $9(13.0)$ \\
\hline Tumor-involved bone & $6(8.7)$ \\
\hline Other & $13(18.8)$ \\
\hline Posttraumatic hydrocephalus & $14(20.3)$ \\
\hline \multicolumn{2}{|l|}{ Attending neurosurgeon } \\
\hline 1 & $39(56.5)$ \\
\hline 2 & $10(14.5)$ \\
\hline 3 & $11(15.9)$ \\
\hline 4 & $8(11.6)$ \\
\hline 5 & $1(1.5)$ \\
\hline Plastic surgery involvement & $22(31.9)$ \\
\hline PEEK implant & $28(40.6)$ \\
\hline HTR-PMI implant & $41(59.4)$ \\
\hline Prior PEEK failures & $6(8.7)$ \\
\hline Prior HTR-PMI failures & $9(13.0)$ \\
\hline Total previous PEEK or HTR-PMI failures & $15(21.7)$ \\
\hline $\begin{array}{l}\text { Mean time elapsed btwn previous cranioplasty attempt } \\
\quad \& \text { new implant surgery } \pm \text { SD (mos) }\end{array}$ & $17.4 \pm 25.7$ \\
\hline Mean postop bone gap in $\mathrm{mm} \pm \mathrm{SD}$ & $3.0 \pm 2.3$ \\
\hline Bone gap $>6 \mathrm{~mm}$ & $6(8.7)$ \\
\hline Mean follow-up in mos \pm SD & $33.3 \pm 32.9$ \\
\hline Total implant failures & $15(21.7)$ \\
\hline Infection rates $\mathrm{w} /$ custom implants & $9(13.0)$ \\
\hline
\end{tabular}

* Data are presented as number (\%) or number (range) unless otherwise indicated.

for 9 implants (16.7\%). There were a total of 41 HTR-PMI implants (59.4\%) and 28 PEEK implants (40.6\%). The average postoperative bone gap was $3 \mathrm{~mm}(\mathrm{SD} \pm 2.3)$, and in 6 of 69 custom cranioplasty cases $(8.7 \%)$ there was a bone gap $>6 \mathrm{~mm}$. The plastic surgeons were involved in 22 cases $(31.9 \%)$. Nine infections after implant placement (13.0\%) were counted as implant failures, because the implants were then discarded. The median patient follow-up was 17.0 months ( \pm 32.8 months). None of these patients were lost to follow-up.

\section{Univariate Analysis}

A univariate analysis was conducted to evaluate all of the variables and compare them with the outcome of implant failure (Table 2). A large postoperative bone gap had a nonsignificant trend toward implant failure with a mean gap of $4.5 \mathrm{~mm}$ in failed cases versus 2.6 in successful implantations $(\mathrm{p}=0.10)$. When dichotomized between those with a gap $>6 \mathrm{~mm}$ and those with a gap $<6 \mathrm{~mm}$ between the implant and the frontal bone, those with bone gaps $\geq 6 \mathrm{~mm}$ were more likely to experience implantation failure $(p=0.02)$. Unexpectedly, a prior failure of a PEEK or HTR-PMI implant was associated with a higher probability of implant success $(p=0.03)$. A shorter time from craniectomy or bone flap resorption to implantation had a near-significant association with implant failure $(\mathrm{p}=0.08)$. Patient age, race, sex, reason for custom cranial implant, attending surgeon, plastic surgery involvement were not associated with implant failure according to univariate analysis (Table 2), nor were a prior history of autologous cranioplasty, the presence of a previous infection, or the length of time since the infection (data not shown). Our main predictor of interest was the type of implant used (PEEK or HTR-PMI), and neither product had a statistically significant advantage over the other $(\mathrm{p}=0.96)$.

\section{Multivariate Analysis}

Using multivariate logistic regression, we developed a predictive model. The significant variables on univariate analysis (bone gap $>6 \mathrm{~mm}$ and prior PEEK implant/HTRPMI failure) were tested with the model, as were the nearsignificant variable (decreased time since the last implant failure) and the primary research question of implant type (PEEK vs HTR-PMI; Table 3). A bone gap between the implant and the native frontal bone $(\mathrm{p}=0.03$, OR $8.3,95 \%$ CI 1.2-55.9) was found to be the only independent risk factor for implant failure.

\section{Implant Type Selection (PEEK vs HTR-PMI)}

We looked not only at the failure rate of each implant type but also at what factors influenced the choice of which implant type to use (PEEK or HTR-PMI; Table 4). According to the univariate analysis, PEEK implants were used more frequently when the mechanism of original need for cranioplasty was traumatic $(p=0.02)$, when there was posttraumatic hydrocephalus present $(\mathrm{p}=0.01)$, or when there was a shorter time since the initial event or previous cranioplasty attempt $(p=0.004)$. In terms of surgeon preferences, one of the attending neurosurgeons was much more likely to choose HTR-PMIs, 2 others preferentially used PEEK, while 2 others split their preferences $(p=0.002)$. PEEK was used more commonly when plastic surgeons were involved in the case as co-surgeons ( $\mathrm{p}=$ 0.01 ). The patient age, the complication rate (infection and CSF leak), and a postoperative bone gap $>6 \mathrm{~mm}$ were not associated with the type of implant chosen.

\section{Discussion}

We present a retrospective cohort study of 54 pediatric patients who had 69 implantations of either PEEK or HTR-PMI custom cranial implants. The overall implant failure rate was $21.7 \%$, and there was no association between implant type (PEEK vs HTR-PMI) and failure rate. Univariate analysis of risk factors showed that patients with a gap $>6 \mathrm{~mm}$ between the implant and the frontal bone were more likely to have an implant failure, while those patients with a prior custom cranioplasty failure (PEEK or HTR-PMI) were actually less likely to experience implant failure. Multivariate analysis of significant and near-significant univariate variables indicated that a 
TABLE 2. Primary univariate analysis with implant failure as the outcome*

\begin{tabular}{|c|c|c|c|}
\hline Variable & Implant Failure $(n=15)$ & Implant Success $(n=54)$ & $p$ Value \\
\hline Age (yrs), mean $\pm S D$ & $6.6 \pm 5.9$ & $8.5 \pm 5.3$ & 0.23 \\
\hline Males & $11(73.3)$ & $39(72.2)$ & 0.93 \\
\hline Caucasian race & $14(93.3)$ & $47(87.0)$ & 0.68 \\
\hline Etiology & & & 0.86 \\
\hline Trauma & $9(60.0)$ & $32(59.3)$ & \\
\hline Infected craniotomy flap & $3(20.0)$ & $6(11.1)$ & \\
\hline Tumor-involved bone & $1(6.7)$ & $5(9.3)$ & \\
\hline Other & $2(13.3)$ & $11(20.3)$ & \\
\hline Posttraumatic hydrocephalus & $4(26.7)$ & $10(18.5)$ & 0.49 \\
\hline Attending neurosurgeon & & & 0.29 \\
\hline 1 & $7(46.7)$ & $32(59.3)$ & \\
\hline 2 & $3(20.0)$ & $7(13.0)$ & \\
\hline 3 & $3(20.0)$ & $8(14.8)$ & \\
\hline 4 & $1(6.7)$ & $7(13.0)$ & \\
\hline 5 & $1(6.7)$ & $0(0)$ & \\
\hline Plastic surgery involvement & $6(40.0)$ & $16(29.6)$ & 0.42 \\
\hline Prior failure of PEEK or HTR-PMI & $0(0.0)$ & $15(27.8)$ & 0.03 \\
\hline $\begin{array}{l}\text { Mean time elapsed btwn previous cranioplasty attempt \& new } \\
\text { implant surgery } \pm \text { SD (mos) }\end{array}$ & $10.8 \pm 11.1$ & $19.4 \pm 28.4$ & 0.08 \\
\hline Custom cranial implant & & & 0.96 \\
\hline PEEK & $6(40.0)$ & $22(40.7)$ & \\
\hline HTR-PMI & $9(60.0)$ & $32(59.3)$ & \\
\hline Mean bone gap \pm SD $(\mathrm{mm})$ & $4.5 \pm 3.9$ & $2.6 \pm 2.1$ & 0.10 \\
\hline Bone gap $>6 \mathrm{~mm}$ & $4(26.7)$ & $2(3.7)$ & 0.02 \\
\hline
\end{tabular}

* Data are presented as number (\%) or number (range) unless otherwise indicated.

bone gap of $>6 \mathrm{~mm}$ between the implant and the frontal bone was the only independent risk factor for implant failure.

PEEK has been used for some time in orthopedic and spinal surgery. ${ }^{11}$ It is stronger than bone and compares favorably with titanium in biomechanical testing yet does not have thermal conduction as a limitation. ${ }^{7,11}$ However, PEEK does not integrate with the native bone flap through bony fusion and therefore must rely on a binding fibrotic reaction and tight approximation to the bone flap. ${ }^{11}$ The literature regarding PEEK cranioplasties is scant, consisting of 3 small case series of 12, 6, and 4 patients, respectively, in addition to scattered case reports. ${ }^{6,8,11}$ Although it is quite difficult to draw conclusions with these small numbers with limited follow-up, the combined complication rate is approximately $33 \%$. Nevertheless, the reports

TABLE 3. Multivariate logistic regression analysis with implant failure as the outcome

\begin{tabular}{lcc}
\hline \multicolumn{1}{c}{ Variable } & OR $(95 \% \mathrm{Cl})$ & $\mathrm{p} \mathrm{Value}$ \\
\hline Implant type (PEEK) & $0.50(0.13-2.00)$ & 0.33 \\
\hline Prior implant failure & $\mathrm{NS}$ & 0.96 \\
\hline $\begin{array}{l}\text { Time elapsed btwn previous cranioplasty } \\
\text { attempt \& new implant surgery }\end{array}$ & $1.05(0.98-1.12)$ & 0.16 \\
\hline Bone gap $>6 \mathrm{~mm}$ & $8.3(1.2-55.9)$ & 0.03 \\
\hline
\end{tabular}

NS = not significant. highly recommend PEEK implants as a cranioplasty option when the native bone is unavailable, because of the reduced operative time and technical ease of placement. $6,8,11$ Still, no long-term follow-up has been reported.

The HTR-PMI is composed of a nonresorbable porous polymer of several synthetic materials with specific surface properties of increased porosity and hydrophilic negative charge designed to encourage bony incorporation and growth while repelling bacterial infection. ${ }^{14}$ The cranioplasty literature regarding HTR-PMIs is also small, but the material has been used extensively in dentoalveolar reconstructions for over 40 years..$^{10}$ Four HTR-PMI cranioplasty series consisted of $<20$ patients each without long-term follow-up. No complications were reported in some series, and a rate of $18 \%$ was reported in one series. ${ }^{4,5,10,14}$ A more recent series of 21 patients reported a complication rate of almost $25 \%$; prior infection, smoking, and diabetes were all not associated with increased complications. ${ }^{10}$

\section{Implant Type}

The total of 41 HTR-PMIs and 28 PEEK implants makes this the largest reported series of each type of cranioplasty implant in the literature. The primary question of this study was to determine which implant type was the most successful for repairing cranial defects. We found that these implants were essentially equivalent with respect to their success rates $(p=0.96)$. This is an impor- 
TABLE 4. Univariate analysis with implant type (PEEK vs HTR-PMI) as the outcome*

\begin{tabular}{|c|c|c|c|}
\hline Variable & PEEK & HTR-PMI & $\mathrm{p}$ Value \\
\hline No. of implants & 28 & 41 & \\
\hline Median age \pm SD (yrs) & $7.4 \pm 5.0$ & $8.6 \pm 5.8$ & 0.35 \\
\hline Males & $24(85.7)$ & $27(65.9)$ & 0.17 \\
\hline Caucasian race & $25(89.3)$ & $37(90.2)$ & 0.71 \\
\hline $\begin{array}{l}\text { Mean time elapsed btwn previous } \\
\text { cranioplasty attempt \& new } \\
\text { implant surgery } \pm \text { SD (mos) }\end{array}$ & $8.3 \pm 7.3$ & $23.8 \pm 31.6$ & 0.004 \\
\hline \multicolumn{4}{|l|}{ Etiology } \\
\hline Trauma & $23(82.1)$ & $18(43.9)$ & 0.02 \\
\hline Infected craniotomy flap & $3(10.7)$ & $6(14.6)$ & \\
\hline Tumor-involved bone & $1(3.6)$ & $5(12.2)$ & \\
\hline Other & $1(3.6)$ & $12(29.3)$ & \\
\hline \multicolumn{4}{|l|}{ Attending neurosurgeon } \\
\hline 1 & $9(32.1)$ & $30(73.2)$ & 0.002 \\
\hline 2 & $7(25.0)$ & $3(7.3)$ & \\
\hline 3 & $4(14.3)$ & $7(17.1)$ & \\
\hline 4 & $7(25.0)$ & $1(2.4)$ & \\
\hline 5 & $1(3.6)$ & $0(0)$ & \\
\hline Plastic surgery involvement & $14(50.0)$ & $8(19.5)$ & 0.01 \\
\hline Posttraumatic hydrocephalus & $10(35.7)$ & $4(9.8)$ & 0.01 \\
\hline Implant complications & & & 0.54 \\
\hline No complication & $24(85.7)$ & $33(80.5)$ & \\
\hline Infected implant & $4(14.3)$ & $5(12.2)$ & \\
\hline CSF hygroma/leak & $0(0.0)$ & $1(2.4)$ & \\
\hline $\begin{array}{l}\text { Postop implant adjustment (not } \\
\text { replacement) }\end{array}$ & $0(0.0)$ & $2(4.9)$ & \\
\hline Previous custom implant failure & $5(17.9)$ & $10(24.4)$ & 0.57 \\
\hline Bone gap $>6 \mathrm{~mm}$ & $3(10.7)$ & $3(7.3)$ & 0.68 \\
\hline
\end{tabular}

* Data are presented as number (\%) or number (range) unless otherwise indicated.

tant contribution since these two common implants have never been directly compared. The overall failure rate of $21.7 \%$ is consistent with the recent reports on HTR-PMI and PEEK cranioplasty implants. ${ }^{10,11}$

\section{Time Elapsed Between Previous Cranioplasty Attempt and New Implant Surgery}

The time elapsed between previous cranioplasty attempt and new implant surgery approached significance $(p=0.08)$ in the univariate analysis but was not significant in the multivariate analysis $(\mathrm{p}=0.16)$. Although we did not find it to have an independent association with implant failure, this trend warrants further discussion. One hypothesis is that the shorter time between craniectomy or resorption and implant placement may be a reflection of more infections causing implant failures, as some literature suggests ${ }^{13,15}$ that early cranioplasty after craniectomy may predispose to infection. An alternative hypothesis is that the shorter interval may not permit the entire bone remodeling process to occur. This may hinder successful implant placement, because a bone gap may develop due to the native bone continuing to be resorbed and to remodel, thus precluding a tight native bone and implant fusion.

\section{Prior Failure of PEEK or HTR-PMI and Prior Infection}

One important and interesting finding from this study is that prior PEEK or HTR-PMI implant failure was associated with subsequent implant success $(p=0.03)$; there were no implant failures for any of the 15 implants that were placed after a previous PEEK or HTR-PMI implant failure. Furthermore, previous infection did not predispose patients to an increased risk of failure or infection $(p=0.72)$. We are unsure why prior failure predisposed patients to successful subsequent reimplantation; however, our best hypothesis is that, after reimplantation, more time has passed since the initial injury (e.g., infection or trau$\mathrm{ma}$ ), and the surrounding native bone has had more time to complete its healing (i.e., further resorption). Therefore, increased subsequent bone gaps are less likely upon reimplantation, because the bone is more quiescent and not in active remodeling and thus is more favorable for healing with implantation. The ramifications of these findings are significant, as subsequent surgical salvage procedures are usually significantly less successful than the original attempts, especially in the setting of a previous infection. . $^{3,9,12}$ This finding can give surgeons confidence in attempting a second custom PEEK or HTR-PMI implantation, even after a previous failure from infection.

\section{Bone Gap}

We found that an increased bone gap $>6 \mathrm{~mm}$ was an independent risk factor for implant failure (OR 8.3, 95\% CI $1.2-55.9, \mathrm{p}=0.03)$. The implant failure rate for a bone gap of $>6 \mathrm{~mm}$ was $66.7 \%$ versus $17.5 \%$ when the bone gap was $<6 \mathrm{~mm}$. These findings are especially important in this surgical series, because bone gap width is a modifiable risk factor based on surgical technique. This suggests that taking the extra surgical time to achieve a near-perfect approximation of the native bone with the custom cranial implants may be critical to successful implantation. The majority of patients with a bone gap $>6$ $\mathrm{mm}$ were 5 years of age and younger (4/6 patients, $66.7 \%$ ). We have previously documented younger age as a risk factor for bone flap resorption after autologous cranioplasty. It seems logical that a bone gap in a younger child would be harder to integrate with a custom implant, because the head grows more rapidly in younger children compared with the head of a teenager or older child with near-complete head growth.

\section{Surgeon Selection of Implant Type}

It is interesting to note the fairly large number of significant variables that were associated with the use of PEEK implant or HTR-PMI as the custom cranial implant. Diverse factors including traumatic etiology, who was involved in surgery, time since previous cranioplasties, and presence of hydrocephalus were all statistically significant, illustrating the specific situations and personal preferences that highlight the need for further study. If further trials confirm our findings that the two implants have similar outcomes, surgeon preference will be an appropriate criterion for selecting the specific implant. 


\section{Limitations}

The principal limitation of this study is that it is a retrospective cohort study and not a prospective trial. Another limitation is that the small number of clinical events (15 failures) is a small sample size for the number of variables analyzed. Therefore, we recommend that our conclusions serve as hypothesis generators for further prospective study of the importance of eliminating bone gaps with custom cranial implantation and not as definitive guidelines.

\section{Conclusions}

The pediatric patients in our study who underwent custom cranioplasty faced an implant failure rate of $21.7 \%$ with initial custom PEEK or HTR-PMI cranial implants. No significant difference was found in implant failure with either implant type (PEEK or HTR-PMI). Multivariate analysis of risk factors revealed that a bone gap $>6 \mathrm{~mm}$ was an independent risk factor for implant failure. We recommend that a tight approximation of the implant to the native bone be achieved in all custom cranial implant procedures to provide a greater opportunity for success. Fortunately, if custom cranioplasty with a PEEK implant or HTR-PMI fails, revision custom cranioplasty with a PEEK or HTR-PMI procedure appears to have an extremely high success rate.

\section{Acknowledgment}

We thank Kristin Kraus, MSc, for editorial assistance preparing this paper.

\section{References}

1. Bowers CA, Riva-Cambrin J, Hertzler DA II, Walker ML: Risk factors and rates of bone flap resorption in pediatric patients after decompressive craniectomy for traumatic brain injury. J Neurosurg Pediatr 11:526-532, 2013

2. Chim H, Schantz JT: New frontiers in calvarial reconstruction: integrating computer-assisted design and tissue engineering in cranioplasty. Plast Reconstr Surg 116:1726-1741, 2005

3. Duddy JC, Allcutt D, Crimmins D, O'Brien D, O'Brien DF, Rawluk D, et al: Foramen magnum decompression for Chiari I malformation: a procedure not to be underestimated. Br J Neurosurg 28:330-334, 2014

4. Eppley BL: Craniofacial reconstruction with computer-generated HTR patient-matched implants: use in primary bony tumor excision. J Craniofac Surg 13:650-657, 2002

5. Eppley BL, Kilgo M, Coleman JJ III: Cranial reconstruction with computer-generated hard-tissue replacement patientmatched implants: indications, surgical technique, and longterm follow-up. Plast Reconstr Surg 109:864-871, 2002
6. Hanasono MM, Goel N, DeMonte F: Calvarial reconstruction with polyetheretherketone implants. Ann Plast Surg 62:653-655, 2009

7. Lethaus B, Safi Y, ter Laak-Poort M, Kloss-Brandstätter A, Banki F, Robbenmenke C, et al: Cranioplasty with customized titanium and PEEK implants in a mechanical stress model. J Neurotrauma 29:1077-1083, 2012

8. Lethaus B, Ter Laak MP, Laeven P, Beerens M, Koper D, Poukens J, et al: A treatment algorithm for patients with large skull bone defects and first results. J Craniomaxillofac Surg 39:435-440, 2011

9. Mazur MD, Sivakumar W, Riva-Cambrin J, Jones J, Brockmeyer DL: Avoiding early complications and reoperation during occipitocervical fusion in pediatric patients. J Neurosurg Pediatr 14:465-475, 2014

10. Nassiri N, Cleary DR, Ueeck BA: Is cranial reconstruction with a hard-tissue replacement patient-matched implant as safe as previously reported? A 3-year experience and review of the literature. J Oral Maxillofac Surg 67:323-327, 2009

11. Ng ZY, Nawaz I: Computer-designed PEEK implants: a peek into the future of cranioplasty? J Craniofac Surg 25: e55e58, 2014

12. Parker SL, Godil SS, Zuckerman SL, Mendenhall SK, Tulipan NB, McGirt MJ: Effect of symptomatic pseudomeningocele on improvement in pain, disability, and quality of life following suboccipital decompression for adult Chiari malformation type I. J Neurosurg 119:1159-1165, 2013

13. Piedra MP, Ragel BT, Dogan A, Coppa ND, Delashaw JB: Timing of cranioplasty after decompressive craniectomy for ischemic or hemorrhagic stroke. J Neurosurg 118:109-114, 2013

14. Roberson JB, Rosenberg WS: Traumatic cranial defects reconstructed with the HTR-PMI cranioplastic implant. J Craniomaxillofac Trauma 3:8-13, 1997

15. Schuss P, Vatter H, Marquardt G, Imöhl L, Ulrich CT, Seifert V, et al: Cranioplasty after decompressive craniectomy: the effect of timing on postoperative complications. J Neurotrauma 29:1090-1095, 2012

\section{Author Contributions}

Conception and design: Riva-Cambrin. Acquisition of data: Bowers, McMullin, Brimley, Etherington. Analysis and interpretation of data: Bowers, McMullin. Drafting the article: Bowers, McMullin. Critically revising the article: Riva-Cambrin, Bowers, Siddiqi. Reviewed submitted version of manuscript: all authors. Approved the final version of the manuscript on behalf of all authors: RivaCambrin. Statistical analysis: Riva-Cambrin.

\section{Correspondence}

Jay Riva-Cambrin, Division of Pediatric Neurosurgery, Department of Neurosurgery, University of Utah, Primary Children's Medical Center, 100 N. Medical Dr., Salt Lake City, UT 841131100. email: jay.riva-cambrin@hsc.utah.edu. 